\title{
Multilinguales
}

\section{La recherche de l'inconnu dans les textes d'Isabelle Eberhardt : la valorisation du mouvement}

The search for the unknown in Isabelle Eberhardt's texts: valorization of the movement

$$
\text { البحث عن المجهول في نصوص ايزابيل ابيرهارد : تثمين الحركة }
$$

\section{Alba Dellavedova}

\section{OpenEdition}

\section{Journals}

\section{Édition électronique}

URL : http://journals.openedition.org/multilinguales/350

DOI : 10.4000/multilinguales.350

ISSN : 2335-1853

\section{Éditeur}

Université Abderrahmane Mira - Bejaia

\section{Référence électronique}

Alba Dellavedova, « La recherche de l'inconnu dans les textes d'Isabelle Eberhardt : la valorisation du mouvement », Multilinguales [En ligne], 8| 2017, mis en ligne le 01 juin 2017, consulté le 17 septembre 2019. URL : http://journals.openedition.org/multilinguales/350; DOI : 10.4000/multilinguales.350

Ce document a été généré automatiquement le 17 septembre 2019.

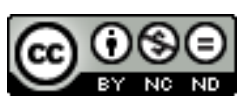

Multilinguales est mise à disposition selon les termes de la Licence Creative Commons Attribution -

Pas d'Utilisation Commerciale - Pas de Modification 4.0 International 


\section{La recherche de l'inconnu dans les textes d'Isabelle Eberhardt : la valorisation du mouvement}

The search for the unknown in Isabelle Eberhardt's texts: valorization of the movement

$$
\text { البحث عن المجهول في نصوص ايزابيل ابيرهارد : تثمين الحركة }
$$

Alba Dellavedova

\section{De l'étude biographique à l'analyse des textes}

1 Isabelle Eberhardt est une écrivaine et une voyageuse d'origine russe qui est née à Genève en 1877 et qui a décidé de quitter l'Europe pour s'installer au Maghreb. Son choix est définitif: Eberhardt adopte les coutumes et les traditions arabes arrivant jusqu'au point de se convertir à l'Islam, probablement autour du 1897. Le voyage eberhardtien n'est pas une simple exploration mais il s'insère dans une dimension existentielle plus vaste. En fait, il implique notre écrivaine d'une manière absolue, sa participation au monde arabe étant extrêmement profonde. Les territoires qu'elle explore sont : la côte tunisienne (de Tunis à Monastir), les principales villes algériennes (Alger, Constantine, Batna, Biskra, Ténès), les anciennes Orléansville et Philippeville (aujourd'hui Chlef et Skikda), les régions du Souf (El Oued, Touggourt, Guémar) de l'Oranie et du Sud oranais. Celle-ci est une zone de guerre, caractérisée par la présence des dissidents algériens. Ici notre écrivaine réalise un reportage.

Isabelle Eberhardt est une auteur connue dans le domaine littéraire et surtout dans celui de la littérature de voyage, mais les raisons sur lesquelles sa célébrité se base sont souvent limitatives de la valeur philosophique-littéraire de sa démarche et de ses textes. Dans les faits, les études biographiques prennent le pas sur des réflexions à caractère textuel. Cette situation peut être expliquée soit par l'originalité du style de vie d'Eberhardt soit par le caractère excentrique de sa personnalité. De plus, la 
détermination de la version authentique de ses textes est extrêmement problématique, ce qui pourrait avoir favorisé davantage l'affirmation d'une approche biographique. L'histoire éditoriale des ouvrages eberhardtiens demeure compliquée et confuse : le premier éditeur, Victor Barrucand, a corrigé les manuscrits et il a conditionné la plupart des publications suivantes. Un travail de reconstruction et de réédition a été accompli par plusieurs chercheurs, parmi lesquels il y a Marie-Odile Delacour, Jean René Huleu, Danièle Masse, Mohamed Rochd. Ceux-ci ont consulté et étudié les manuscrits d'Eberhardt, qui sont conservés aujourd'hui aux Archives nationales d'outre-mer d'Aix-en-Provence. D'ailleurs, l'importance d'une recherche de type textuel est relevée par Mohamed Rochd. Celui-ci affirme que ce sont les textes qui " présentent le plus d'intérêt » parce qu'ils ont « une véritable valeur littéraire » ainsi qu' » une valeur historique réelle » (M. Rochd 1992). Les ouvrages d'Eberhardt sont : des notes de voyage, des reportages (dont le plus important est réalisé dans la région du Sud oranais), des nouvelles et des romans (Rakhil et Trimardeur. Celui-ci est inachevé). Une partie de ces textes a été publiée dans des journaux français et algériens dont l'Athénée de France, le Progrès de l'Est, la Dépêche algérienne, l'Akhbar, Les nouvelles d'Alger.

\section{Les interprétations traditionnelles}

3 Parmi les sujets traditionnellement abordés dans les études biographiques dédiées à Isabelle Eberhardt, il y a la quête identitaire, le déguisement et le nomadisme. En particulier, la démarche eberhardtienne est présentée comme l'exemple d'une renonciation complète aux facteurs identitaires hérités à la naissance (comme le sexe, la culture, le style de vie). Plus généralement, l'écrivaine est proposée comme une femme qui refuse la culture européenne et qui la remplace par la culture arabe, dans le cadre d'une recherche continue de l'Inconnu et du Divers. En effet, Isabelle Eberhardt voyage au Maghreb habillée en homme, sous le nom de Mahmoud Saadi, un jeune taleb tunisien. De plus, elle utilise souvent dans ses écrits des pseudonymes, qui sont indifféremment masculins ou féminins. Ce qui émerge est une totale indifférence envers sa propre identité et son sexe: dans les journaux intimes - publiés premièrement par René-Louis Doyon pour la Connaissance dans les années '20 du dernier siècle - Eberhardt utilise la première personne, mais le moi dont elle se sert, est soit masculin soit féminin. La question du travestissement assume une importance théorique plus vaste lorsqu'on observe qu'Eberhardt déclare vouloir aller au Maghreb pour "devenir quelqu'un» (I. Eberhardt 1988: 308), et s'y créer "une âme, une conscience, une intelligence, une volonté » (I. Eberhardt $1988: 321)$ : « cette dure vie du Désert, un peu moins fatigante puisque je ne serai pas obligé de veiller dans la nuit, achèvera mon éducation d'homme d'action, cette éducation spartiate qui est une arme indispensable dans ma position » (I. Eberhardt $1988:$ 319).

4 Le déguisement n'aurait pas une fonction purement pratique, mais il renverrait à un cadre plus complexe. En ce qui concerne le nomadisme, notre écrivaine se définit à plusieurs reprises comme une nomade, et cet appellatif est utilisé par tous ses biographes. Ceux-ci arrivent jusqu'au point de définir une sorte de nomadisme anthropophile. Par exemple, René-Louis Doyon - un autre éditeur des textes eberhardtiens, qui a publié ses journaux intimes dans une version qui sera reprise dans toutes les publications suivantes - définit Eberhardt par l'expression de la bonne nomade ${ }^{2}$. 


\section{Une réévaluation de la quête identitaire et du nomadisme}

5 Ces deux questions prédominantes - celle de la quête identitaire associée au déguisement et celle du nomadisme - méritent d'être traitées plus en profondeur. De plus, elles devraient aller de pair avec une réflexion herméneutique conduite à partir des textes eberhardtiens et avec une analyse ponctuelle de ses voyages. En ce qui concerne la quête identitaire, la démarche d'Isabelle Eberhardt prévoit effectivement la formation et la création de sa propre personnalité. Cependant, la rapidité avec laquelle ce processus est traditionnellement associé au désir de contact avec l'autre que soimême - considéré ici dans un sens premièrement culturel - et au refus de ses origines européennes, est susceptible d'induire en erreur. Dans les faits, si on fait référence aux textes écrits par Eberhardt, on voit que l'écrivaine n'explique pas son attraction pour le Maghreb comme la conséquence d'une opposition ressentie envers l'Europe. Au contraire, elle affirme un droit individuel et positif, qui est celui de choisir sa culture d'appartenance et le lieu où l'on veut vivre. Voici ce qu'Isabelle Eberhardt écrit au directeur du journal algérien la Dépêche algérienne au propos de sa conversion à l'Islam :

Afin de ne pas passer pour une émule du Dr Grenier ou pour une personne revêtant un costume et s'affublant d'une étiquette religieuse dans un but intéressé quelconque, je tiens à déclarer ici, que je n'ai jamais été chrétienne, que je ne suis pas baptisée et que, quoique sujette russe, je suis musulmane depuis fort longtemps. Ma mère, qui appartenait à la noblesse russe, est morte à Bône, en 1897, après s'être faite musulmane et a été enterrée dans le cimetière arabe de cette ville. (I. Eberhardt, $1988: 328$ ).

6 Derrière ces mots il y a avant tout une affirmation de liberté. Celle-ci est présente dans plusieurs passages des textes eberhardtiens. Notamment, dans cette même lettre, notre écrivaine interpelle directement le directeur du journal et l'interroge par les mots suivants : "À qui cela peut-il nuire, que je préfêre l'horizon onduleux et vague des dunes grises à celui du boulevard ? (I. Eberhardt, $1988:$ 398). La quête identitaire ne passe pas par un refus de la culture européenne, mais elle exprime plutôt l'attraction vers la culture arabe. Par conséquent, l'importance attribuée au sens d'étrangeté devrait être réduite. En effet, bien qu'Isabelle Eberhardt voyage souvent, elle veut s'intégrer dans le monde arabe et partager la culture locale. Même avant son premier départ pour l'Algérie, la jeune Isabelle lit le Coran, étudie la langue arabe et le dialecte kabyle. La recherche d'une extranéité culturelle est remplacée par celle que Catherine Stoll-Simon définit comme une forme "inversée et par conséquent subversive, d'assimilation " (C. Stoll-Simon, 2006 : 42). Ce point de vue, qui pourrait être mis en discussion en raison de son statut quelque peu ethnocentrique, demeure généralement valide. Mohamed Rochd utilise une expression plus centrée définissant Isabelle Eberhardt comme une maghrébine d'adoption et il approfondit la question de l'altérité observant que «l'autre n'est plus alors l'étranger, mais simplement autrui, celui avec lequel on partage un certain nombre de valeurs communes» (M. Rochd, $1991: 10$ ).

D'une manière similaire, le nomadisme eberhardtien devrait être revu à la lumière d'autres considérations. Avant tout, s'il est vrai qu'Isabelle Eberhardt voyage sans interruption, il faut néanmoins observer que ses déplacements sont faits dans des régions connues et que l'Algérie reste le pays privilégié. Cette continuité va de pair avec la tentative d'installation définitive au Maghreb et elle évolue en un désir de 
sédentarité. Eberhardt manifeste souvent la volonté d'interrompre ses pérégrinations, et dans ses journaux intimes elle exprime le désir «d'aller là-bas, à Ouargla, au seuil du grand océan de mystère qui est le Sahara et s'y fixer pour "fonder ce foyer qui de plus en plus " (I. Eberhardt, 1988: 320) lui manque. Eberhardt revient à plusieurs reprises sur cette question : «je crois aussi, ou plutôt je commence à croire que j'ai aussi trouvé mon port (...). Le bonheur domestique est trouvé et, loin de diminuer, semble se raffermir de jour en jour... " (Ibid. : 350). Ces déclarations pourraient être interprétées comme issues d'un moment de faiblesse ou comme le résultat d'une crise temporaire, mais il faudrait néanmoins remarquer le fait que les phrases susmentionnées sont situées à côté d'autres phrases qui exaltent la vie nomade. Cela nous fait croire que le nomadisme eberhardtien est atypique et qu'il ne prévoit pas la négation complète d'une certaine forme de sédentarité.

\section{La description d'un espace en mouvement}

Dans le cas d'Isabelle Eberhardt, l'expérience du Divers n'est pas issue de l'extranéité culturelle, mais elle est le résultat de la richesse du paysage observé. Celui-ci se compose de plusieurs facteurs qui interagissent entre eux d'une manière variée. La diversité consiste dans les produits de cette interaction, qui sont à chaque fois nouveaux. Par conséquent, les éléments qui constituent le paysage qui sera décrit dans les textes d'Eberhardt, se modifient constamment, et cela permet d'avoir une expérience du milieu qui est toujours nouvelle. Cette possibilité est donnée à la fois à l'observateur et au lecteur, qui, comme observe Rachel Bouvet « assiste au dévoilement des multiples facettes d'un même espace » (R. Bouvet et M. Marcil-Bergeron, 2013). La diversité mise en évidence par Isabelle Eberhardt, renvoie à une méthode d'observation proche de celle de l'impressionnisme, mais elle finit par impliquer tous les existants : les habitants, la nature, les objets. La dimension religieuse et celle culturelle participent, par leurs représentations concrètes, à la construction d'un espace en mouvement. C'est ce qui arrive dans le cas suivant :

La mosquée d'El Morkad et son minaret carré, tout proches, semblaient aussi déserts que les ruines environnantes...

Tout à coup, au-dessus de ma tête, un volet de bois s'ouvrit et claqua violemment contre le mur... Un jet de lumière rougeâtre glissa le long de la muraille et vint ensanglanter le pavé... C'était le mueddine qui se levait. (I. Eberhardt, 1988 : 34)

Dans ce passage du texte, on assiste à la véritable manifestation de la religion islamique, qui est ici représentée par la mosquée et par la voix du muezzin. Les paysages qu'Eberhardt décrit sont le résultat d'une interaction complexe qui implique plusieurs facteurs, parmi lesquels il y a le peuple, les traditions, les composantes culturelles et celles religieuses. La nouveauté de la vision est assurée à l'observateur par la variété de relations qui se tressent parmi des éléments divers: "en effet", explique l'écrivaine dans un passage de ses journaux intimes daté du 2 juillet 1902, "jamais deux êtres - l'exception peut-être existe - n'ont vu le même paysage, le même pays de la même façon, sous le même jour, sous la même couleur » (Ibid. : 449). 


\section{Le rôle de l'écriture} tout, c'est à travers ce processus que l'auteur prend conscience de l'avancement de son parcours d'intégration. Dans ses textes, Eberhardt reconstruit un espace vécu, constitué par une interaction à laquelle l'écrivaine a participé. Le milieu agit sur le corps et sur l'esprit d'Eberhardt, lui procurant des sensations, des impressions et des visions, trois mots qui ont une signification précise, qui renvoie à des formes et des degrés de relation différents. D'ailleurs, la vivacité du milieu est soulignée par plusieurs artifices linguistiques et littéraires, parmi lesquels figurent les phrases nominales. Celles-ci font en sorte que les choses apparaissent avant qu'elles soient vues ou perçues par l'observateur. De plus, les composantes du milieu sont souvent les sujets des phrases, ce qui témoigne de leur puissance d'action. Nous rapportons ci-dessous quelques exemples : les deux premiers démontrent l'abondance de phrases nominales, tandis que le troisième présente l'interaction qui se réalise parmi les composantes du milieu. « $\mathrm{Au}$ bout d'une immense plaine désolée, semée de tombes, semblable à celle de Tarzout, des murailles grises, et, dominant tout, un immense palmier solitaire... » (Ibid. : 86).

Sousse, une ville arabe, tortueuse et charmante, étagée sur une colline haute, enclose encore d'une muraille sarrasine, crénelée et d'une blancheur neigeuse. Sur le versant de la colline, en dehors des remparts, des cimetières immenses, entourés de haies de figuiers de Barbarie, brulés et jaunis par le soleil. Plus haut encore, les toits rouges et les bâtiments longs et bas du camp des tirailleurs (Ibid. : 48).

Parfois un burnous passe, se faufile, disparait dans la cour, burnous blanc de M'sili, burnous bleu de deïra...Alors l'une des idoles se lève avec un grand cliquetis de bijoux, et suit le visiteur dans l'ombre chaude des cellules pauvres.

Et M'Sila s'endort ainsi, maraboutique et prostituée, assoupie et ardente, dans la lourde chaleur de la nuit. Les benadir, les vieilles cantilènes religieuses et les sonnailles des bracelets des Ouled-Naïl la bercent doucement (Ibid. : 114).

\section{La multiplicité linguistique}

Dans un cadre théorique ainsi défini, le facteur linguistique joue un rôle fondamental, surtout dans la réception des textes. En effet, Isabelle Eberhardt écrit des œuvres en langue française mais elle utilise souvent des mots arabes. La graphie arabe est parfois maintenue dans les manuscrits - comme le démontre l'édition des textes suivie par Marie-Odile Delacour et Jean René Huleu et publiée par Grasset en 1988-1989 - mais le plus souvent les mots arabes sont proposés en traduction alphabétique française, ce qui témoigne du désir de l'écrivaine d'être lue et comprise par des lecteurs européens francophones. Après avoir réalisé une analyse des champs sémantiques auxquels les termes arabes appartiennent, ce qui ressort est la volonté de sauvegarder les composantes essentielles des paysages décrits, à savoir le caractère spécifique d'habitants, de lieux et d'objets qui sont typiquement arabes. Le lecteur réalise à travers les textes eberhardtiens, une expérience unique, qui consiste dans la perception d'une étrangeté à laquelle il doit se familiariser immédiatement. D'autant plus que les mots arabes sont situés au milieu du texte et que leur compréhension est parfois fondamentale pour celle du récit. Par exemple, les combattants de la région oranaise et leur habillement, sont définis de la manière suivante: "se mêlant au soleil, goumiers montés sur de petits chevaux maigres, tout en muscles, mokhazni en longs burnous noirs brodés de rouge sur la poitrine" (I. Eberhardt, 1988: 128), le territoire situé à la frontière du Maroc se trouve "au-dessus de l'oued à sec qui, pour les mokhazni, représente la hadada, la problématique frontière du Maroc » où « une brise légère court 
sur le sable fin, bruissant à peine dans le drinn et les jujubiers épineux » (Ibid. : 137). Cela survient aussi quand on s'occupe de la religion islamique, comme dans le cas suivant (les mots arabes sont surlignés) :

Le marabout, sans descendance mâle, désigna pour lui succéder après sa mort son unique enfant, qu'il avait instruite en arabe comme le meilleur des tolba. Il préparait à sa fille un rôle bien différent de celui qui incombe généralement à la femme arabe, et c'est elle qui, aujourd'hui, dirige la zaouïya et les khouan, affiliés de la confrérie. (Ibid. : 122)

13 En premier lieu, l'utilisation de ces mots est à même de mettre en évidence la progressive augmentation des connaissances de l'écrivaine sur le milieu ainsi que l'avancement de son intégration. La présence de paroles arabe est particulièrement fréquente dans le reportage réalisé au Sud oranais, qui est caractérisé aussi par la reproduction de prières et de chants traditionnels, comme le suivant :

Les chefs nous annoncent une expédition lointaine :

Mon cœur est mon avertisseur,

Il m'annonce une mort prochaines.

Qui me verra mourir? Qui priera pour moi?

Qui fera pour ma mémoire l'aumône sur ma tombe?

Ah! Qui sait ce que me réserve la destinée de Dieu!

Ma gazelle blanche m'oubliera,

Un autre montera ma douce cavale...

O cœur, tais-toi ! Ne pleure pas, mon œil!

Car les larmes ne servent à rien.

Nul n'obtiendra ce qui n'était pas écrit,

Et ce qui est écrit, nul ne l'évitera...

Calme-toi, mon âme, jusqu'à ce que Dieu ait pitié,

Et si tu ne parviens pas à te calmer, il y a la mort... (Ibid. : 166).

14 D'ailleurs, l'utilisation des mots arabes fait en sorte que la culture et le milieu locaux se manifestent d'une manière directe et immédiate.

\section{Conclusions théoriques}

La lecture attentive des textes reste fondamentale pour la mise en place d'une étude plus consciente de la démarche eberhardtienne et pour la valorisation de ses enjeux théoriques et conceptuels. Les écrits méritent d'être pris en compte avant tout dans leur valeur historique, politique et sociale. En effet, ils contiennent des informations sur la situation existante en Algérie en époque coloniale, qui ont été recueillies au cours de l'enquête du terrain réalisée par l'écrivaine. C'est ce que souligne Edmonde CharlesRoux, l'une des principales biographes d'Eberhardt, qui affirme à ce propos: " connaissez-vous une Française ou une étrangère allée en Algérie faire un véritable travail d'ethnologue ? (...). Elle est en avance de 100 ans sur son temps » (E. Charles-Rou, $1995: 10$ ). La valeur littéraire des œuvres devrait également être mise en relief. Le rapport qui unit Isabelle Eberhardt et le voyage est complexe et entraîne des réflexions à caractère philosophique qui rapprochent notre écrivaine de l'existentialisme. Elles coexistent avec une étude de type ethnologique qui prévoit le recueil de renseignements sur la situation du Maghreb en époque coloniale. À côté de cela, une participation personnelle et intime existe, et il ne faudrait surtout pas la négliger. Le texte assume dans ce cadre une importance fondamentale : son analyse nous permet d'abord de revoir la véritable signification théorique de la quête identitaire, de la recherche d'Inconnu et du 
nomadisme. D'ailleurs, il joue un rôle assez important dans l'accomplissement du processus de valorisation du monde arabe et il est en même temps le témoignage de l'avancement $d u$ parcours d'intégration réalisé par Eberhardt. Cela conduit à l'utilisation d'artifices linguistiques et littéraires qui permettent de représenter l'interaction parmi les composantes du milieu qui aboutit à la création du paysage. En conclusion, par sa propre expérience, Isabelle Eberhardt invite le lecteur à commencer un voyage qui ne soit pas une exploration casuelle de milieux inconnus. Pourtant, le message fondamental de l'écrivaine est l'affirmation de la libre détermination individuelle, et le sens d'étrangeté qu'elle valorise ne doit pas être interprété dans un sens culturel mais bien existentiel, à savoir comme le résultat de la mutation continue à laquelle chaque espace est soumis. La connaissance du milieu ainsi que le partage culturel demeurent une valeur. Dans ce cadre théorique, la quête identitaire, le déguisement et le nomadisme assument une signification nouvelle et spécifique. La nouveauté de la démarche de notre écrivaine est donc consistante et substantielle, car elle définit un espace ouvert et disponible, qui est à même d'accueillir les nouveaux venus.

\section{BIBLIOGRAPHIE}

BOUVET, Rachel, « Variations autour d'un paysage : le désert chez Isabelle Eberhardt », in Pratique de l'espace en littérature, article d'un cahier Figura. Disponible sur le site de l'observatoire de l'imaginaire contemporain, consulté le 2 février 2017. D'abord paru dans BOUVET Rachel et FOLEY Françoise (éd.), Figura, centre de recherche sur le texte et l'imaginaire, Montréal, coll. « Figura », vol. 7, 2008, p. 105-118. Disponible sur « http://oic.uqam.ca/fr/articles/variationsautour-dun-paysage-le-desert-chez-isabelle-eberhardt ».

BOUVET, Rachel, MARCIL-BERGERON Myriam, « Pour une approche géopoétique du récit de voyage », in Arborescences : revue d'études françaises, $\mathrm{n}^{\circ} 3$, 2013. Disponible sur « http:// www.erudit.org/fr/revues/arbo/2013-n3-arbo0733/1017364ar/».

CHARLES-ROUX, Edmonde, Nomade j'étais, les années africaines d'Isabelle Eberhardt (1899-1904), Grasset, Paris, 1995.

CHARLES-ROUX, Edmonde, Un désir d'Orient : jeunesse d'Isabelle Eberhardt (1877-1899), Grasset, Paris 1995.

EBERHARDT, Isabelle Écrits sur le sable, œuvres complètes I, édition établie, présentée et annotée par Marie-Odile Delacour et Jean René Huleu, Grasset, Paris, 1988.

EBERHARDT, Isabelle, Écrits sur le sable, œuvres complètes II, édition établie, présentée et annotée par Marie-Odile Delacour et Jean René Huleu, Grasset, Paris, 1989.

EBERHARDT, Isabelle, Mes journaliers, précédés par La vie tragique de la bonne nomade, par René-Louis Doyon, Paris, La connaissance, coll. « Les textes, 4 », 1923.

FORGEAU, Catherine, « Du récit ethnologique à la fiction romanesque. Pour une "mise en œuvre" de la réalité », in L’homme et la société, année 1939, vol. 134, n 4, p. 45-62. 
HADOUCHE-DRIS, Louise, « L'œuvre algérienne d'Isabelle Eberhardt, une écriture à revisiter », in Insaniyat, 46, 2009, p. 45-50, mis en ligne le 11 avril 2012, consulté le 2 février 2017. Disponible sur: « http://insaniyat.revues.org/180».

HEDI, Abdel-Jaouad, «Isabelle Eberhardt: Portrait of the Artist as a Young nomad », in Yale French Studies. Post/Colonial Conditions : Exiles, Migrations and Nomadism, vol. 2, Yale, 1993, p. 93-117. Disponible sur « https://www.jstor.org/stable/2930089 ?seq =1\#page_scan_tab_contents ». Isabelle Eberhardt, coll. 20.10, Centre culturel algérien, Paris.

Littérature et espaces, actes du XXXe Congrès de la Société Française de Littérature Générale et Comparée, SFLGC, Limoges 20-22 septembre 2001, Presses universitaires de Limoges, Limoges, 2003.

Littératures et temps colonial. Métamorphoses du regard sur la Méditerranée et l'Afrique, actes du colloque d'Aix-en-Provence. 7-8 avril 1997, éd. par Jean-Robert Henry, Lucienne Martini HENRY Jean-Robert, Centre des Archives d'Outre-Mer, Aix-en-Provence, Édisud, coll. « Mémoires méditerranéennes », 1999.

REZZOUG Simone, «État présent des travaux sur Isabelle Eberhardt ». Disponible sur « http:// www.mmsh.univ-aix.fr».

ROCHD, Mohamed, Isabelle Eberhardt, le dernier voyage dans l'ombre chaude de l'Islam, Entreprise nationale du livre, Alger, 1991.

ROCHD, Mohamed, Isabelle, une maghrébine d'adoption, préface par Jean Déjeux, Office des publications universitaires, Alger 1992.

Série X - dons et acquisitions 1758/1956, 23 X Fonds Isabelle Eberhardt, Archives nationales d'outre-mer, Aix-en-Provence.

STOLL-SIMON, Catherine, Si Mahmoud ou la renaissance d'Isabelle Eberhardt, éditions Tarik, coll « Zellige », Léchelle, 2006.

\section{NOTES}

2. René-Louis Doyon accompagne l'édition des journaux intimes d'Isabelle Eberhardt par une préface intitulée La vie tragique de la bonne nomade.

\section{RÉSUMÉS}

Isabelle Eberhardt est une écrivaine-voyageuse, protagoniste d'une exploration du territoire algérien réalisée entre la fin du XIX et le début du XX ${ }^{\mathrm{e}}$ siècle. La démarche eberhardtienne est intéressante en raison de sa complexité. En effet, le voyage d'Eberhardt prévoit une étude ethnologique du milieu, une activité d'écriture et un processus formatif qui consiste dans la création de sa propre personnalité. Ces objectifs sont poursuivis au Maghreb à travers une participation complète : la jeune Isabelle adopte les coutumes et les traditions arabes, elle se convertit à l'Islam, elle parle l'arabe et elle fréquente quotidiennement les habitants de la région. 
À travers la lecture de ses textes, des concepts tels que le nomadisme, la quête identitaire et la recherche du Divers assument une signification précise. En particulier, le discours identitaire doit être mis en relation avec la forte affirmation de sa propre libre détermination individuelle : plus que renoncer à l'Europe, Isabelle Eberhardt choisit l'Afrique. Cette liberté s'élargit de la dimension géographique à celle culturelle. Le nomadisme en revanche aboutit souvent à l'exploration de lieux bien connus : il se rapproche dans ce sens d'un mouvement conscient, fait par quelqu'un qui est à même d'apprécier les changements continus du paysage.

Isabelle Eberhardt is a travel writer who explores the Maghreb from 1897 up to 1904. Academic and professional researches have always concentrated on the biography of this eccentric woman, which has contributed to the creation of a legendary figure that goes by the name of Isabelle Eberhardt. The complexity of the process makes it intersting: Eberhardt's journey provides for an ethnological study of the environment as well as the writing and a personal training, that consists in the creation of her own personality. These aims are pursued in the Maghreb through a total participation: the young Isabelle adopts arabic customs and traditions, she converts to Islam, she speaks Arabic and she spends her time with the inhabitants of the region. Thanks to the study of her texts, some concepts, such as nomadism, the quest of identity and the research of the Different, assume a specific meaning. Particularly, the identity matter should be related to the strong affirmation of her own free individual determination: more than renouncing to Europe, Isabelle Eberhardt chooses Africa. This kind of freedom extends to a geographic and cultural dimension. For example, nomadism usually interests well-known places: it reflects a conscious movement done by someone who can appreciate the constant mutation of the landscape.

landscape, Isabelle Eberhardt, female Travel, Maghreb, unknown

\section{INDEX}

Mots-clés : paysage, Isabelle Eberhardt, voyage au féminin, Maghreb, inconnu

مناظر الطبيعة, إيزابيل إبيرهارد, رحلة نسويه, المغرب, المجهول فهرس الكلمات المفتاحية:

\section{AUTEUR}

\section{ALBA DELLAVEDOVA}

Université Paris IV Sorbonne

Università degli Studi di Milano 